\title{
How Women Evaluate Syndromic Recurrent Urinary Tract Infections
}

\author{
Woong Bin Kim, Sang Wook Lee, Kwang Woo Lee, Jun Mo Kim, Young Ho Kim, Min Eui Kim \\ Department of Urology, Soonchunhyang University Bucheon Hospital, Soonchunhyang University College of Medicine, Bucheon , Korea
}

Purpose: To investigate the clinical manifestations of patients with the principal complaint of syndromic recurrent urinary tract infection (UTI), correlate these symptoms with the results of urine cultures, and identify the characteristics that can be used to differentiate UTI from similar diseases.

Materials and Methods: A total of 212 consecutive patients with syndromic recurrent UTIs over a 24 month period were evaluated. The major symptoms were recorded using the UTISA questionnaire and VAS. The patients were divided into group $A(n=98$; positive urine and urethral swab cultures) and group $B(n=114$; negative cultures), and the symptoms were compared. For group B, cystoscopy was used to diagnose 61 patients who complained of pain levels $\geq 6$ on the VAS.

Results: The proportion of patients with the classic symptoms of UTI (dysuria, urinary frequency, lower abdominal discomfort during bladder filling, and urgency) was similar in groups A and B. Significantly more patients complained of urethral pain in group B, and significantly fewer patients had gross hematuria, low back pain, a post-voiding sensation of residual urine, and general symptoms compared to group A. Of the 61 patients with a VAS $\geq 6,29,28$, and four were diagnosed with bladder pain syndrome, interstitial cystitis, and urethral pain syndrome, respectively.

Conclusions: In patients with syndromic recurrent UTI, the classic symptoms were not sufficiently characteristic to allow bacterial cystitis to be differentiated from other bladder diseases. Diagnostic cystoscopy and VAS can assist in making a differential diagnosis in patients with non-bacterial syndromic recurrent UTIs.

Keywords: Urinary tract infections; Cystitis; Interstitial cystitis

Copyright (c) 2019, Korean Association of Urogenital Tract Infection and Inflammation. All rights reserved. (s) This is an open access article distributed under the terms of the Creative Commons Attribution (c) (1) (s) Non-Commercial License (http://creativecommons.org/licenses/by-nc/4.0) which permits unrestricted non-commercial use, distribution, and reproduction in any medium, provided the original work is properly cited.
Received: 1 April, 2019

Revised: 23 May, 2019

Accepted: 28 June, 2019
Correspondence to: Young Ho Kim

(iD) https://orcid.org/0000-0003-3959-0928

Department of Urology, Soonchunhyang University Bucheon Hospital, 170 Jomaru-ro, Wonmi-gu, Bucheon 14584, Korea

Tel: +82-32-621-5058, Fax: +82-32-621-5018

E-mail: yhkuro@schmc.ac.kr

\section{INTRODUCTION}

Bacterial cystitis is a very common disease in females; 7 million are diagnosed with acute cystitis annually in the United States alone [1]. Recurrent infections are encountered in $25-50 \%$ of patients with acute cystitis [2]. The classic symptoms of acute cystitis include pain and lower urinary tract problems, such as dysuria, hematuria, and the need for frequent urination. Patients with recurrent cystitis re- peatedly present with the symptoms of sporadic acute cystitis, requiring frequent hospital visits. Although the practices vary geographically, it is common to diagnose and treat such diseases based on the clinical symptoms because a urine culture takes time. In real practice, empirical antibiotics are prescribed before a urine culture is performed, and if the symptoms subside, a urine culture is often not ordered. Therefore, it is often difficult to differentially diagnose urinary tract infection (UTI) from other diseases when 
a patient visits a hospital with the symptoms of recurrent UTI. For this reason, few studies have addressed the symptomatic differential diagnosis of recurrent UTI from other bladder diseases with similar symptoms. In particular, it is difficult to make an accurate diagnosis when no bacteria are detected in the urine culture when patients are given empirical antibiotics prior to culture or when they complain of atypical cystitis symptoms. Some lower urinary tract symptoms of nonbacterial bladder diseases, such as interstitial cystitis, bladder pain syndrome, and urethral pain syndrome, are similar to the symptoms of urinary tract bacterial infections, and it is likely that the former conditions are diagnosed late or diagnosed mistakenly as bacterial cystitis. This may result in the inappropriate use of antibiotics, adversely affecting the patient quality-of-life. This study investigated the clinical manifestations of patients with the principal complaint of syndromic recurrent UTI, correlated these symptoms with the results of a urine culture, and identified the characteristics that can be used to differentiate UTI from similar diseases.

\section{MATERIALS AND METHODS}

This prospective and consecutive observational study examined 212 females aged $49.6 \pm 14.8$ years with syndromic recurrent UTIs regardless of the results of urine culture, and who were referred to or visited the authors' research institute in the 24 months prior to commencing the study in January 2015. The study included patients with histories of at least two symptoms similar to those of acute cystitis at least three times over the last year, or at least twice over the last 6 months, again regardless of the urine culture results [3]. Each patient enrolled complained of at least one of the pain symptoms of acute cystitis (dysuria, lower abdominal discomfort, and low back pain), and one other symptom of the Korean version of the UTI symptom assessment (UTISA) standardized questionnaire [4]. The seven symptoms of the UTISA are urinary frequency, urgency, dysuria, a sense of incomplete emptying, lower abdominal discomfort, low back pain, and hematuria [5]. The exclusion criteria were a prescription of antibiotics in other hospitals in the two weeks prior to presentation at the authors' institute, a history of catheter use in the one month prior, any specific disease that could cause urination-related symptoms (an anatomical or congenital abnormality; a neurogenic bladder; diabetes; stroke; large post void residual urine volume $>100 \mathrm{ml}$; a gynecological disease, such as endometriosis, pelvic organ prolapse, pelvic inflammatory disease, vaginitis, interstitial cystitis, bladder pain syndrome, and complicated UTI; and/or infection of any other organ, including the upper urinary tract or upper respiratory tract). Major symptoms were recorded using the UTISA questionnaire $[4,5]$ and the symptoms not included in the questionnaire were recorded separately. In addition, the pain or discomfort level was measured using a visual analogue scale (VAS). Urine was collected using catheters and urinalysis, and a urine culture was performed. On urinalysis, each urine specimen was examined directly under a microscope and pyuria was considered to be present when $\geq 5$ white blood cells per high power field were evident. Urine specimens were inoculated onto blood agar and MacConkey agar plates, and any bacterial count $>10^{5}$ $\mathrm{CFU} / \mathrm{ml}$ was considered to indicate bacteriuria. In addition, Ureaplasma urealyticum and Mycoplasma hominis were cultured from urethral swabs using the MYCOFAST ${ }^{\circledR}$ Evolution 2 system (International Microbio, Signes, France), and the culture positivity rates were examined. All patients were divided into group A ( $\mathrm{n}=98,46.2 \%$; positive on any culture) and group $B(n=114,53.8 \%$; negative); the symptoms of the two groups were compared (Fig. 1). In group A, the uropathogens causing cystitis were examined. Extendedspectrum $\beta$-lactamase (ESBL)-positive organisms, which are antibiotic-resistant, were analyzed. In group B (with negative urine cultures), differential diagnoses were performed for 61 cases $(53.5 \%)$ who complained of pain levels $\geq 6$ on the VAS on their first visit. All patients underwent diagnostic cystoscopy that was performed by the same physician to determine the cause of the pain. Using the definition of the International Continence Society (ICS), those who complained of discomfort in the suprapubic area associated with bladder filling and had a need to urinate frequently during both the day and night, but lacked any specific lesion evident on cystoscopy, were diagnosed with bladder pain syndrome [6,7]. In addition, using the National Institute of Diabetes and Digestive and Kidney Diseases (NIDDK) criteria, patients were considered to have interstitial cystitis if they exhibited diffuse glomerulation or were confirmed to have a Hunner's lesion in the bladder when evaluated cystoscopically under anesthesia, and who had a bladder capacity $<350 \mathrm{ml}$ [8]. Those who lacked specific bladder 


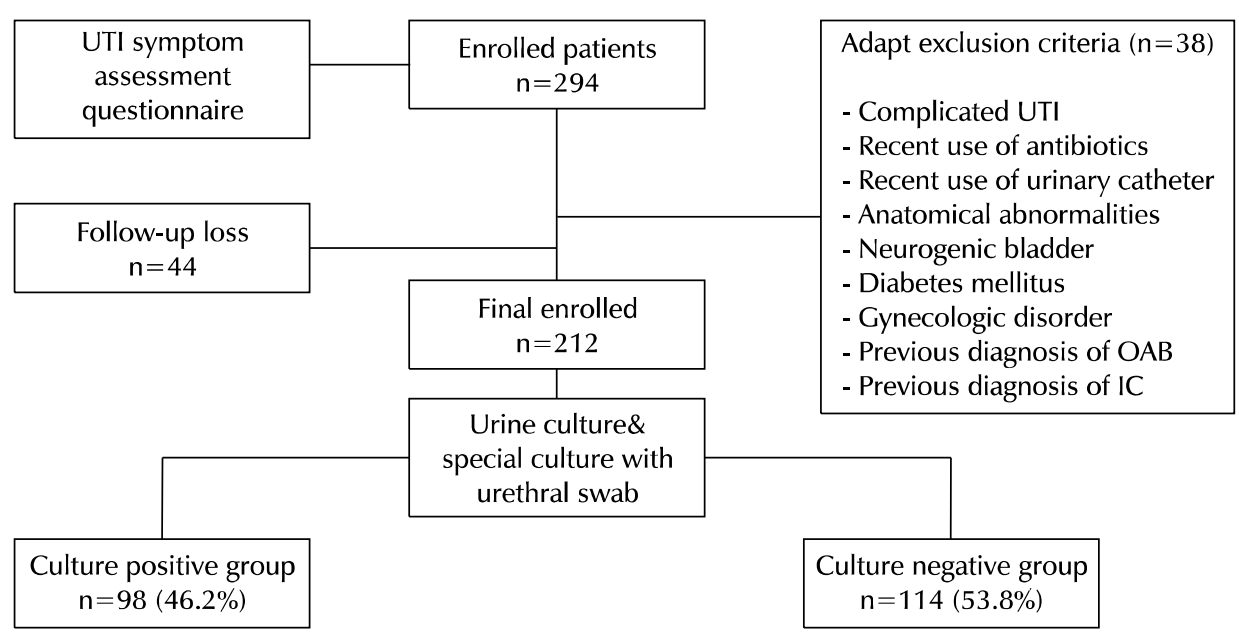

Fig. 1. Flowchart used for inclusion in the study. UTI: urinary tract infection, OAB: overactive bladder, IC: interstitial cystitis.

Table 1. Clinical characteristics and symptoms of patients with syndromic recurrent urinary tract infections according to the urine culture results

\begin{tabular}{lcccc} 
& \multirow{2}{*}{ Variable } & Total $(\mathrm{n}=212)$ & \multicolumn{2}{c}{ Results of urine culture } \\
\cline { 3 - 4 } & & Bacterial $(\mathrm{n}=98)$ & Non-bacterial $(\mathrm{n}=114)$ & $\mathrm{p}$-value \\
\hline Age $(\mathrm{y})$ & $49.6 \pm 14.8$ & $50.2 \pm 15.7$ & $49.2 \pm 14.0$ & 0.617 \\
Number of visit & $6.4 \pm 3.5$ & $4.9 \pm 2.7$ & $7.8 \pm 3.5$ & $<0.05$ \\
Pyuria & $138(65.1)$ & $90(91.8)$ & $24(21.1)$ & $<0.05$ \\
Symptoms & & & & \\
Dysuria & $177(83.5)$ & $84(85.7)$ & $93(81.6)$ & 0.419 \\
$\quad$ Urinary frequency & $159(75.0)$ & $72(73.5)$ & $87(76.3)$ & 0.633 \\
Lower abdominal discomfort during bladder filling & $134(63.2)$ & $60(61.2)$ & $74(64.9)$ & 0.579 \\
Urgency & $83(39.2)$ & $38(38.8)$ & $45(39.5)$ & 0.917 \\
Urethral pain & $73(34.4)$ & $19(19.4)$ & $54(47.4)$ & $<0.05$ \\
Gross hematuria & $49(23.1)$ & $30(30.6)$ & $19(16.7)$ & $<0.05$ \\
Low back pain & $35(16.5)$ & $29(29.6)$ & $6(5.3)$ & $<0.05$ \\
Post-voiding sensation of residual urine & $24(11.3)$ & $18(18.4)$ & $6(5.3)$ & $<0.05$ \\
General symptoms & $67(31.6)$ & $40(40.8)$ & $27(23.7)$ & $<0.05$ \\
\hline
\end{tabular}

Values are presented as mean \pm standard deviation or number $(\%)$.

findings, who had a normal bladder capacity, and who complained only of pain around the urethra, were diagnosed with urethral pain syndrome. Statistical analysis was performed using SPSS version 16.0K for Windows (SPSS, Inc., Chicago, IL, USA). An analysis of the variance and an independent $\mathrm{t}$-test were used for the between-group comparisons. The categorical variables were compared using a Pearson's chi-squared test. p-values $<0.05$ were considered significant.

\section{RESULTS}

\section{Population Characteristics}

The mean age of the 212 patients was $49.6 \pm 14.8$ years and the mean number of hospital visits (to both our hospital and others) with the symptoms of recurrent cystitis was $6.4 \pm 3.5$. The number of patients who had visited hospitals $\leq 3$ times, 4 to 10 times, and $>10$ times was 36 (17.0\%), 136 (64.2\%), and 40 (18.9\%), respectively. The mean ages of the group A ( $n=98,46.2 \%)$ and group B cases $(n=114,53.8 \%)$ were similar at $50.2 \pm 15.7$ and $49.2 \pm 14.0$ years, respectively $(p=0.617)$. The number of hospital visits made by the group A and group B patients was $4.9 \pm 2.7$ and $7.8 \pm 3.5$, respectively; the difference was significant $(\mathrm{p}=0.012)$. Of all 212 patients, pyuria was evident in 138 (65.1\%), of whom 90 (91.8\%) and 24 (21.1\%) were in group A and B, respectively $(\mathrm{p}<0.05)$ (Table 1$)$.

\section{Routine Urine Culture Data}

Of the 212 patients, 81 (38.2\%) yielded bacteria on the routine urine culture. The most common causative organisms of UTI were Escherichia coli (64 cases; 79.0\%) followed by Klebsiella pneumoniae (6 cases; 7.4\%). In addition, four cases grew coagulation-negative Staphylococcus (4.9\%), three 
Table 2. Result of microorganisms the patients with recurrent cystitis in female

\begin{tabular}{|c|c|c|c|c|}
\hline Cultured organism & Data & Routine and special culture $(+)$ & Special culture $(+)$ only & ESBL positive (\%) \\
\hline \multicolumn{5}{|l|}{ Routine urine culture $(n=81)$} \\
\hline Escherichia coli & $64(79.0)$ & & & $6(7.4)$ \\
\hline Klebsiella pneumoniae & $6(7.4)$ & & & $1(1.2)$ \\
\hline Coagulation negative Staphylococcus & $4(4.9)$ & & & \\
\hline Enterococcus faecalis & $3(3.7)$ & & & $1(1.2)$ \\
\hline Proteus spp. & $3(3.7)$ & & & \\
\hline Group B Streptococcus & $1(1.2)$ & & & \\
\hline \multicolumn{5}{|l|}{ Special culture with swab $(n=212)$} \\
\hline Ureaplasma urealyticum & $22(10.4)$ & 9 & 13 & \\
\hline Mycoplasma hominis & $16(7.5)$ & 7 & 9 & \\
\hline$U$. urealyticum $+M$. hominis & $9(4.2)$ & 4 & 5 & \\
\hline
\end{tabular}

Values are presented as number (\%) or number only.

Enterococcus faecalis (3.7\%), three Proteus spp. (3.7\%), and one Group B Streptococcus (1.2\%). Eight patients tested positive for ESBL organisms on culture (9.9\%); six patients grew ESBL (+) E. coli, 1 ESBL (+) $K$. pneumoniae, and 1 ESBL (+) E. faecalis (Table 2).

\section{Culture of Ureaplasma urealyticum and $\mathrm{Myco}^{-}$ plasma hominis}

To examine the causes of frequent bladder irritation, the patients were screened for U. urealyticum and $M$. hominis ("special culture[s]"). Of all 212 patients, 22 (10.4\%) and 16 (7.5\%) were positive for U. urealyticum and $M$. hominis, respectively. Both pathogens were cultured from nine patients (4.2\%). Twelve cases $(5.7 \%)$ were positive on both the routine and special cultures; $U$. urealyticum was observed in nine cases, $M$. hominis in seven, and both pathogens in four. Seventeen patients (8.0\%), who were negative on the routine urine culture but positive on the special urine culture, were finally diagnosed with bacterial cystitis. Thirteen, nine, and five patients tested positive for U. urealyticum, $M$. hominis, and for both pathogens, respectively (Table 2).

\section{Symptoms in Patients with Syndromic Recur- rent UTI}

In all 212 patients, the most common symptom was dysuria (177 cases; $83.5 \%$ ), followed in order by the urinary frequency in 159 (75.0\%), lower abdominal discomfort during bladder filling in 134 (63.2\%), urgency in 83 (39.2\%), urethral pain in $73(34.4 \%)$, gross hematuria in 49 (23.1\%), low back pain in $35(16.5 \%)$, and post-voiding sensation of residual urine in $24(11.3 \%)$ (Table 1$)$.

\section{Comparison of the Symptoms between Those with Bacterial Recurrent UTIs and Non-Bacte- rial Syndromic Recurrent UTIs}

Of the 98 group A patients (46.2\% of the total), who were positive on both the routine and special urine cultures, the most common symptom was dysuria (84 cases; $85.7 \%$ ) followed in order by urinary frequency (72 cases; $73.5 \%$ ), lower abdominal discomfort during bladder filling (60 cases; $61.2 \%)$, urgency in 38 cases (38.8\%), gross hematuria in 30 (30.6\%), low back pain in 29 (29.6\%), urethral pain in 19 (19.4\%), and a post-voiding sensation of residual urine in 18 (18.4\%). In addition, 40 patients (40.8\%) had general symptoms, such as aches, chills, nausea, and general weakness. Of the 114 patients (53.8\%) in group B (negative upon urine culture), the most common symptom was dysuria (93 cases, $81.6 \%$ ). The number of patients complaining of urinary frequency, lower abdominal discomfort during bladder filling, and urgency were 87, 74, and 45 (76.3\%, 64.9\%, and $39.5 \%$ ), respectively. No proportion differed significantly from the group A proportions $(\mathrm{p}=0.419, \mathrm{p}=0.633, \mathrm{p}=0.579$, and $p=0.917$, respectively). The number of patients complaining of urethral pain was 54 , which was proportionally much higher than that of group A ( $19.4 \%$ vs. $47.4 \%, \mathrm{p}<0.05)$. Of all group B patients, 19 (16.7\%), six (5.3\%), and six (5.3\%) had gross hematuria, low back pain, and a post-void sensation of residual urine, respectively. These proportions were all significantly lower than the group A proportions. In addition, only 27 patients (23.7\%) complained of general symptoms; this proportion was significantly lower than that of group A (40.8\%) (Table 1). 


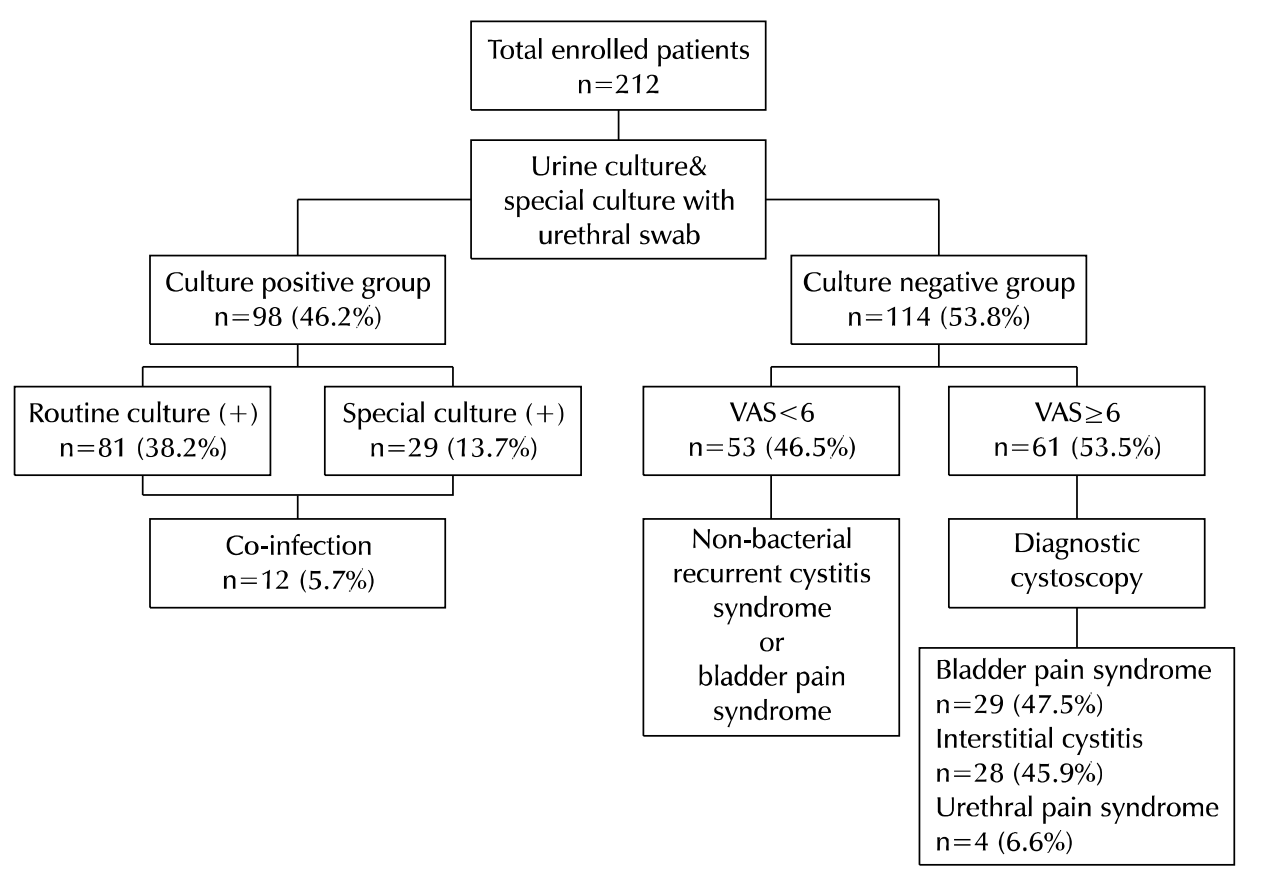

Fig. 2. Urine culture-based classification of patients with syndromic recurrent urinary tract infections. VAS: visual analogue scale.

\section{Classification of Patients with Non-Bacterial Syndromic Recurrent UTIs}

Of the 114 group B patients (53.8\%), who had symptoms of recurrent cystitis without a bacterial infection, diagnostic cystoscopy was performed on 61 (53.5\%) patients who complained of pain at a level $\geq 6$ on the VAS when admitted, and who continued to complain of high-level pain, despite seven days of treatment with empirical antibiotics. Of these 61 patients, 29 (47.5\%) were diagnosed with bladder pain syndrome using the ICS criteria because they did not exhibit ulcers or glomerulation on cystoscopy performed under general anesthesia, although they did complain of discomfort when the bladder was full. They comprised 13.7\% (29/212) of all subjects. The prevalence of the condition among the 114 patients testing negative upon urine culture was $25.4 \%$ (29/114). Finally, 28 (45.9\% of group B) patients who underwent diagnostic cystoscopy were diagnosed with interstitial cystitis; these patients comprised $13.2 \%$ (28/212) of all subjects and $24.6 \%$ (28/114) of those with negative urine cultures. In addition, four patients were diagnosed with urethral pain syndrome (6.6\%), comprising $1.9 \%$ of all patients and $3.5 \%$ of group B patients. Cystoscopy was not performed on 53 patients (46.5\%) who had negative urine cultures and whose VAS pain scores were $<6$. These patients were diagnosed with non-bacterial recurrent cystitis syndrome or bladder pain syndrome and were scheduled for further diagnosis through follow-up testing (Fig. 2).

\section{DISCUSSION}

Recurrent cystitis is defined as cystitis associated with bacterial growth on a urine culture that has occurred at least twice over the last 6 months or at least three times over the last year [3]. Studies of recurrent cystitis based on this definition have focused largely on prevention, risk factors, and self-initiated management, and the disease [9-12]. Those studies included only patients with recurrent cystitis associated with positive urine cultures; few studies have explored the diagnostic processes, or the final diagnoses, of patients with similar symptoms but for whom the urine culture results were unavailable, causing diagnostic confusion [13]. Patients who recurrently visited hospitals with symptoms similar to cystitis, accompanied by pain in the lower urinary tract, were defined as having a "syndromic recurrent UTI" before the urine culture was performed, and their symptoms and the associations between the symptoms and final diagnoses were investigated.

An important feature of the diagnosis of acute cystitis is the fact that patients are often diagnosed based only on their initial clinical symptoms. Bent et al. [14] suggested that when one or more symptoms of lower UTI are evident, the probability of infection is approximately $50 \%$, and when certain symptoms (such as dysuria and urinary frequency, but not vaginal discharge) are combined, the probability of infection reaches 90\%. Komaroff [15] defined acute un- 
complicated cystitis as the acute-onset of dysuria with or without urinary frequency; only symptoms were used in diagnosis. In many cases, acute cystitis is diagnosed and treated based only on the symptoms. Therefore, all principal symptoms were recorded using the Korean version of the UTISA questionnaire [4,5] to make the work more objective. Questionnaires that carefully describe the symptoms of cystitis are both rare and uncommonly used. Therefore, it is often difficult to differentiate between patients with and without infectious diseases. Therefore, the use of an objective questionnaire when diagnosing cystitis may be necessary because an initial differential diagnosis is essential for accurate diagnosis and treatment. In practice, acute cystitis diagnosed only by reference to specific symptoms is simply considered to be an uncomplicated infection of the bladder when the symptoms disappear after a short course of empirical antibiotics. On the other hand, patients who continue to complain of lower urinary tract symptoms may be difficult to diagnose if an acute infection has already been treated, or there is no evidence of re-infection.

In the present study, the positive culture rates of patients with syndromic recurrent UTIs were $38.2 \%$ on the routine urine culture and $13.7 \%$ on the urethral swab special culture of $U$. urealyticum or $M$. hominis. A routine culture was negative but a special culture was positive in 17 of the 212 patients (8.0\%). Potts et al. [16] considered that the two bacterial species mentioned above caused chronic lower urinary tract symptoms, and treated patients with antibiotics to which the organisms were sensitive, resulting in an improvement in symptoms in approximately $91 \%$ of patients. In the present study, the culture rates were relatively high, and a special culture of urethral swabs is likely to be helpful in the diagnosis of patients with syndromic recurrent UTIs. On the other hand, as both microbes are also present in normal urogenital organs [17], it is unclear if they are best regarded as part of the normal flora or uropathogens.

No single theory satisfactorily explains how symptoms develop when the bladder is inflamed by a bacterial infection or otherwise. One widely accepted hypothesis is that the symptoms can be attributable to a primary deficiency in the array of bladder epithelial cells $[18,19]$. Another study [20] reported that upon animal injection with uropathogenic E. coli, the contractile response of the bladder was controlled in the short-term by endothelial nitric oxide synthase, but a long-term contraction of the bladder detrusor muscle was regulated by extracellular signal-regulated kinase 1,2-mediated control of inducible nitric oxide synthase activity. Recently, Arya et al. [21] reported that patients with recurrent cystitis may not have active infections but rather, exhibit bladder oversensitivity. The cited authors compared the filling cystometric data from patients with and without a history of recurrent cystitis, and considered that prior disease may trigger chronic symptoms by causing electrophysiological changes to the unmyelinated C-fibers, which are abundant in the bladder. Therefore, the symptoms are caused by chronic inflammation triggered by prior stimulation, and not present stimulation, such as a bacterial infection. Non-bacterial disease may be caused by a similar mechanism.

Dysuria refers to pain, burning sensation, or discomfort, during urination [22], and is caused by the inflammation of epithelial cells of the urethra. Dysuria frequently develops upon a UTI, to the extent that many clinicians regard dysuria as being caused only by such infection, whereas it can in fact be caused by other conditions [23,24]. In the present study, 177 of the 212 patients complained of dysuria (83.5\%), which is the most common symptom of syndromic recurrent UTIs. Dysuria was the most frequent symptom in both the infected and non-infected group. Hence, the infection status cannot be distinguished by this symptom alone; no betweengroup difference was apparent. This is likely because inflammation per se causes dysuria; the inflammation may or may not be associated with an infection. In addition, the urinary frequency, lower abdominal discomfort during bladder filling, and urgency, which were the next three most common symptoms, are also believed to reflect inflammation per se, and did not differ in extent between the groups. The UTISA questionnaire has the limitation that it does not assess whether the complaints can be attributable to a recurrent bacterial infection or non-bacterial bladder disease. Further diagnostic questionnaires based on more objective criteria will be needed.

The proportion of patients with pain around the urethra, gross hematuria, low back pain, a sense of incomplete emptying, and general symptoms, which are relatively atypical of UTI, differed significantly between the two groups. Low back pain is a common symptom of lower UTI $[10,25]$, and is believed to be associated with the spread of infection to the pelvic cavity and upper urinary tract. Patients with bacterial cystitis complained of low back pain much more 
often than patients with non-bacterial bladder disease, suggesting that in the latter patients, the pain and urinary symptoms were limited to the bladder. Hematuria was also more frequent in patients with bacterial cystitis. In 2001, Gomes et al. [26] reported that 60 out of 148 patients with interstitial cystitis had hematuria, which was gross in two (1.4\%). In the present study, 19 out of 114 (16.7\%) patients with non-bacterial disease complained of gross hematuria compared to only one out of 28 (3.6\%) with interstitial cystitis. Nevertheless, further studies on patients with interstitial cystitis will be needed; the patient numbers were smaller than those of previous studies.

As general symptoms are often non-specific, detailed patient histories were taken. Those suspected of lower or upper UTIs that were complications of other diseases, and whose general symptoms had other causes (such as upper respiratory tract infection) were excluded. This limited the general symptoms complained of during hospital visits to those caused by lower UTIs. Infected patients complained more of general weakness, fever, and chills than noninfected patients did, probably because the bacteria were being disseminated in the blood. On the other hand, further work will be needed because the details of the general symptoms presented were not examined.

Bladder pain syndrome or interstitial cystitis is a disease that lacks a clear pathophysiology; patients often complain of pain caused by chronic bladder inflammation and symptoms similar to those of cystitis, such as urinary frequency and urgency. Indeed, it is difficult to differentiate the symptoms of a lower UTI from non-bacterial bladder disease; no significant difference was observed between the two diseases except in terms of a few atypical symptoms. Therefore, it is essential to identify the disease accurately when a patient presents with symptoms of recurrent UTI and the urine culture is negative.

Braunstein et al. [27] compared the symptoms and characteristics of patients with interstitial cystitis accompanied and not accompanied by ulcers and found no significant between-group difference except in terms of age. This highlights the importance of diagnostic cystoscopy, as opposed to a symptomatic differential diagnosis. In addition, it was difficult to make a differential diagnosis based on the symptoms alone. Hence, diagnostic cystoscopy was performed on patients with negative urine cultures. On the other hand, in practice, it is impossible to perform cystoscopy on all patients. Therefore, the extent of pain was measured using a VAS, which is an internationally accepted measure of self-reported pain. Cystoscopy was performed under local or general anesthesia in 61 patients (53.5\%) who reported continuously high pain scores (VAS $\geq 6$ ) despite the analgesic treatment (nonsteroidal anti-inflammatory drugs, five days) given while awaiting the results of the urine culture.

In 2002, the ICS [6] defined bladder pain syndrome as suprapubic pain associated with bladder filling, accompanied by other symptoms, such as increased day and night urinary frequencies, in the absence of a proven UTI or other obvious pathology. Interstitial cystitis is confirmed when ulcers in the bladder, or diffuse glomerulation of the bladder is evident on cystoscopy, and the strict NIDDK criteria also require that the functional bladder capacity be $<350 \mathrm{ml}$ [8]. As cystitis caused by bacterial infections is not associated with any physical reduction in bladder capacity, the use of the NIDDK bladder capacity criterion was appropriate, and stricter criteria were employed.

The patients were classified into those in whom a cystoscope was difficult to introduce, who did not show specific bladder lesions, and who complained of severe pain around the urethra, as with those with urethral strictures or urethral pain syndrome. Those with negative urine cultures and VAS pain levels $<6$ were classified as having non-bacterial cystitis syndrome or bladder pain syndrome. Diagnostic cystoscopy was negative in these patients, and further follow-up was recommended. Such patients require continued observation and analysis of symptom changes.

Irwin et al. [28] reported that the incidence of recurrent UTI in patients with BPS/IC (bladder pain syndrome/ interstitial cystitis) was only $7.5 \%$, which was similar to that in non-BPS/IC subjects. Thus, as the prevalence of BPS/IC in UTI patients is low, diagnostic cystoscopy was performed on patients with positive urine cultures. In addition, as many patients with positive cultures reported symptom improvement after treatment with empirical antibiotics, and there was no need for cystoscopy in such patients. If, however, interstitial cystitis is accompanied by UTI, a further examination will be needed if a patient has persistent pain or continues to complain of symptoms, even though the urine culture is negative after treatment.

Diagnostic cystoscopy on patients with VAS pain scores $\geq 6$ showed that 28 out of 61 (45.9\%) had interstitial cystitis, comprising $13.2 \%$ of all those with syndromic recurrent UTI. 
This rate is relatively high, and indicates that patients with syndromic recurrent UTIs, who are negative on the urine culture but report repeated or continuous severe pain can be aided by cystoscopy; however, an appropriate pain score cut-off must be defined. Future work should focus on the development of more systematic diagnostic criteria, and on disease classification in females with syndromic recurrent UTIs.

\section{CONCLUSIONS}

In females with recurrent symptoms similar to those of cystitis, the classic symptoms, including dysuria, urgency, and frequency, are not sufficiently characteristic to allow cystitis to be differentiated from other bladder diseases. Pain around the urethra was more frequent in patients with non-bacterial syndromic recurrent UTIs than in patients with bacterial cystitis, whereas gross hematuria, low back pain, a sense of incomplete emptying, and general symptoms were less frequent, indicating that atypical symptoms are more helpful when attempting to differentiate bacterial from non-bacterial disease. Upon diagnostic cystoscopy of the patients with non-bacterial syndromic recurrent UTIs, 28 out of 61 (45.9\%) of those with pain VAS scores $\geq 6$ were diagnosed with interstitial cystitis, suggesting that cystoscopy, in addition to urine culture and VAS evaluation, can be helpful when a differential diagnosis is required for patients with syndromic recurrent UTI.

\section{CONFLICT OF INTEREST}

No potential conflict of interest relevant to this article was reported.

\section{ACKNOWLEDGMENTS}

This study was supported by the Soonchunhyang University Research Fund.

\section{AUTHOR CONTRIBUTIONS}

W.B.K. participated in data collection, performed the statistical analysis and wrote the manuscript. S.W.L., K.W.L., J.M.K. and M.E.K. participated in the study design. Y.H.K. participated in the study design and coordination and helped to draft the manuscript. All authors read and approved the final manuscript.

\section{ORCID}

Woong Bin Kim, https://orcid.org/0000-0002-7369-490X

Sang Wook Lee, https://orcid.org/0000-0001-9660-4092

Kwang Woo Lee, https://orcid.org/0000-0002-8882-2492

Jun Mo Kim, https://orcid.org/0000-0003-4978-1867

Young Ho Kim, https://orcid.org/0000-0003-3959-0928

Min Eui Kim, https://orcid.org/0000-0002-4909-1041

\section{REFERENCES}

1. Hooton TM, Scholes D, Hughes JP, Winter C, Roberts PL, Stapleton $\mathrm{AE}$, et al. A prospective study of risk factors for symptomatic urinary tract infection in young women. N Engl J Med 1996;335:468-74.

2. Mabeck CE. Treatment of uncomplicated urinary tract infection in non-pregnant women. Postgrad Med J 1972;48:69-75.

3. Scholes D, Hooton TM, Roberts PL, Stapleton AE, Gupta K, Stamm WE. Risk factors for recurrent urinary tract infection in young women. J Infect Dis 2000;182:1177-82.

4. Min KS, Kim YH, Kim JM, Shin KL, Hong JY, Kim ME. Development of a Korean version of the urinary tract infection symptoms assessment questionnaire. Korean J Urol 2009;50: 361-8.

5. Clayson D, Wild D, Doll H, Keating K, Gondek K. Validation of a patient-administered questionnaire to measure the severity and bothersomeness of lower urinary tract symptoms in uncomplicated urinary tract infection (UTI): the UTI Symptom Assessment questionnaire. BJU Int 2005;96:350-9.

6. Abrams P, Cardozo L, Fall M, Griffiths D, Rosier P, Ulmsten U, et al. The standardisation of terminology of lower urinary tract function: report from the Standardisation Sub-committee of the International Continence Society. Neurourol Urodyn 2002; 21:167-78.

7. van de Merwe JP, Nordling J, Bouchelouche P, Bouchelouche K, Cervigni M, Daha LK, et al. Diagnostic criteria, classification, and nomenclature for painful bladder syndrome/interstitial cystitis: an ESSIC proposal. Eur Urol 2008;53:60-7.

8. Gillenwater JY, Wein AJ. Summary of the National Institute of Arthritis, Diabetes, Digestive and Kidney Diseases workshop on interstitial cystitis, National Institutes of Health, Bethesda, Maryland, August 28-29, 1987. J Urol 1988;140:203-6.

9. Albert X, Huertas I, Pereiró II, Sanfélix J, Gosalbes V, Perrota C. Antibiotics for preventing recurrent urinary tract infection in non-pregnant women. Cochrane Database Syst Rev 2004;(3): CD001209.

10. Fihn SD. Clinical practice. Acute uncomplicated urinary tract 
infection in women. N Engl J Med 2003;349:259-66.

11. Gupta K, Hooton TM, Roberts PL, Stamm WE. Patient-initiated treatment of uncomplicated recurrent urinary tract infections in young women. Ann Intern Med 2001;135:9-16.

12. Stapleton A. Prevention of recurrent urinary-tract infections in women. Lancet 1999;353:7-8.

13. Gupta K, Trautner BW. Diagnosis and management of recurrent urinary tract infections in non-pregnant women. BMJ 2013; 346:f3140.

14. Bent S, Nallamothu BK, Simel DL, Finn SD, Saint S. Does this woman have an acute uncomplicated urinary tract infection? JAMA 2002;287:2701-10.

15. Komaroff AL. Acute dysuria in women. N Engl J Med 1984; 310:368-75.

16. Potts JM, Ward AM, Rackley RR. Association of chronic urinary symptoms in women and Ureaplasma urealyticum. Urology 2000;55:486-9.

17. Ingham HR, MacFarlane WV, Hale JH, Selkon JB, Codd AA. Controlled study of the prevalence of $\mathrm{T}$ strain mycoplasmata in males with non-gonococcal urethritis. Br J Vener Dis 1966; 42:269-71.

18. Rajasekaran M, Stein P, Parsons CL. Toxic factors in human urine that injure urothelium. Int J Urol 2006;13:409-14.

19. Parsons CL, Forrest J, Nickel JC, Evans R, Lloyd LK, Barkin J, et al. Effect of pentosan polysulfate therapy on intravesical potassium sensitivity. Urology 2002;59:329-33.
20. Weng TI, Chen WJ, Wu HY, Liu SH. Uropathogenic Escherichia coli alters muscle contractions in rat urinary bladder via a nitric oxide synthase-related signaling pathway. J Infect Dis 2006; 194:1774-82.

21. Arya LA, Northington GM, Asfaw T, Harvie H, Malykhina A. Evidence of bladder oversensitivity in the absence of an infection in premenopausal women with a history of recurrent urinary tract infections. BJU Int 2011;110:247-51.

22. Seller RH. Differential diagnosis of common complaints. 3rd ed. Philadelphia: Saunders; 1996. p. 341-52.

23. Bremnor JD, Sadovsky R. Evaluation of dysuria in adults. Am Fam Physician 2002;65:1589-96.

24. Kurowski K. The women with dysuria. Am Fam Physician 1998;57:2155-64, 2169-70.

25. Tanagho EA, McAninch JW. Smith's general urology. 16th ed. New York: McGraw-Hill; 2004. p. 203-27.

26. Gomes CM, Sánchez-Ortiz RF, Harris C, Wein AJ, Rovner ES. Significance of hematuria in patients with interstitial cystitis: review of radiographic and endoscopic findings. Urology 2001; 57:262-5.

27. Braunstein R, Shapiro E, Kaye J, Moldwin R. The role of cystoscopy in the diagnosis of Hunner's ulcer disease. J Urol 2008; 180:1383-6.

28. Irwin P, Samsudin A. Reinvestigation of patients with a diagnosis of interstitial cystitis: common things are sometimes common. J Urol 2005;174:584-7; discussion 587. 